Erratum

\title{
Production and nitrogen responses of the African dwarf shrub Indigofera spinosa to defoliation and water limitation
}

\section{M.B. Coughenour, J.K. Detling, I.E. Bamberg, and M.M. Mugambi}

Oecologia (1990) 83:546-551

Due to an unfortunate error, part of the legends for Figures 1-5 were omitted.

Legend for Figures 1 and 4 should appear as follows:

roots; stems; litter; leaf; spine; $\square$ flower

Legend for Figures 2 and 5 should appear as follows:

$\square$ roots; stems; 